\title{
Hearing Impairment and Hearing Aid (HA) Usage in Singapore
}

Tan Tock Seng H O S P I T A L $\overline{\text { National Healthcare Group }}$
Eu Chin $\mathrm{Ho}^{1}$, HanYu Zhang ${ }^{2}$, Warren Ming Wu Ong ${ }^{2}$, KeXin $\mathrm{Li}^{2}$, Yen Tze Eileen Bei ${ }^{1}$, Satya Vijay Reddy Medapati $^{3}$, Aruni Seneviratna ${ }^{4}$

\section{Background}

Singapore have a population of 5.6 million, with estimated prevalence of disabling hearing loss (HL) of $9.6 \%$, while HA penetration rate is only at $3.3 \%$.

\section{Objectives}

To examine the epidemiological and audiological profile, pattern of HA fitting and usage in Singapore.

\section{Methods}

Retrospective cross-sectional study. 1068 subjects issued with HAs at a tertiary hospital between 2001 and 2013.

\section{Number (\%)}

Total number of subjects fitted with HA included in study $1068(100.0)$

Number of subjects with $1^{\text {st }}$ time HA fitting only $990(92.7)$

Number of subjects with $1^{\text {st }}$ time and successive HA fitting $78(7.3)$

Table 1. Summary of subjects fitted with HA

\section{$\underline{\text { Results }}$}

Mean age was 70 years with $50.8 \%$ male. Mean hearing loss (HL) was $63.1 \mathrm{~dB}$ and $69.5 \%$ had at least moderate-severe HL.

Sensorineural HL was present in $66.4 \%$ and $69.8 \%$ had symmetrical HL.

'Behind-the-Ear' HAs were cheaper than 'InEar' HAs. HA was used $\geq 4$ days/week by $85.6 \%$ but $>7$ hours per day by only $35.7 \%$.

Only $18 \%$ received bilateral first HA fitting.

\begin{tabular}{|c|c|c|}
\hline Age at $1^{\text {st }}$ HA fitting, years (SD) & Mean & 70 (13) \\
\hline Initial HL (PTA aided ear), dB (SD) & Mean & $63.1(13.9)$ \\
\hline \multirow[t]{5}{*}{ Initial HL severity of aided ear ${ }^{1}, \mathrm{n}(\%)$} & Mild & $40(3.7)$ \\
\hline & Moderate & $286(26.8)$ \\
\hline & Moderate-Severe & 465 (43.5) \\
\hline & Severe & $240(22.5)$ \\
\hline & Profound & $37(3.5)$ \\
\hline \multirow[t]{3}{*}{ Baseline type of $\mathrm{HL}, \mathrm{n}(\%)$} & Sensorineural & $708(66.4)$ \\
\hline & Mixed and Conductive & $172(16.1)$ \\
\hline & Combined type & $187(17.5)$ \\
\hline \multirow[t]{2}{*}{ HL Symmetry, n (\%) } & Symmetrical & $745(69.8)$ \\
\hline & Asymmetrical & $323(30.2)$ \\
\hline Total HA cost ${ }^{2}$, SGD (SD) & \multicolumn{2}{|c|}{ Mean 2104.63 (1703.67) } \\
\hline HA cost per Unit ${ }^{2}, \mathrm{SGD}(\mathrm{SD})$ & \multicolumn{2}{|c|}{ Mean $1688.77(1194.55)$} \\
\hline HA cost per Fitting ${ }^{2}$, SGD (SD) & Mean & 1898.09 (1554.4) \\
\hline \multirow[t]{3}{*}{ Regularity of Usage, $n$ (\%) } & $\geq 4$ days per week & $675(85.6)$ \\
\hline & $\leq 3$ days per week & $114(14.4)$ \\
\hline & Total responders & $789(100.0)$ \\
\hline \multirow[t]{4}{*}{ Daily Usage, n (\%) } & $<4 \mathrm{~h}$ & 251 (31.9) \\
\hline & $4-7 h$ & 255 (32.4) \\
\hline & $>7 \mathrm{~h}$ & $281(35.7)$ \\
\hline & Total responders & $787(100.0)$ \\
\hline \multirow{8}{*}{$\begin{array}{l}\text { HA type, } n(\%) \\
\text { (Total number of all HAs fitted, } 1 \text { st time and } \\
\text { successive) } \\
\text { Total missing data }=1(0.1 \%)\end{array}$} & Total number of $\mathrm{HA}$ fitted & $1383(100.0)$ \\
\hline & Standard BTE & $928(67.1)$ \\
\hline & RIC & $49(3.5)$ \\
\hline & Total BTE HA types & 977 (70.6) \\
\hline & ITE & $90(6.5)$ \\
\hline & ITC & 164 (11.9) \\
\hline & $\mathrm{CIC}$ & $152(11)$ \\
\hline & Total In-Ear HA types & 406 (29.4) \\
\hline
\end{tabular}

1 Mild ( $\leq 40 \mathrm{~dB}$ ); Moderate (>40-55 dB); Moderate-Severe (>55-70 dB); Severe (>70-90 dB); Profound (>90 dB)

2 Hearing aid cost, availability of data $94.5 \%$

Younger age and symmetrical $\mathrm{HL}$ were predictors of bilateral HA uptake.

\begin{tabular}{lrccc} 
& & Unilateral HA & Bilateral HA & p value \\
\hline Total Users, $n(\%)$ & Number & $876(82.0)$ & $192(18.0)$ & - \\
\hline Age at HA first fitting, years & Mean (SD) & $71(13)$ & $69(15)$ & 0.119 \\
\hline \multirow{2}{*}{ Symmetry, $\mathrm{n}(\%)$} & Symmetrical & $584(66.7)$ & $161(83.9)$ & $<0.001$ \\
& Asymmetrical & $292(33.3)$ & $31(16.1)$ & \\
\hline
\end{tabular}

Table 3. Comparison of subjects with Unilateral 1st HA fitting vs. Bilateral 1st HA fitting

While better hearing of aided ear and $>7$ hours of daily HA usage were predictors of successive HA fitting.

\section{Conclusion}

Singapore HA users were elderly and with advanced HL. Bilateral HA adoption and average daily use were low.

Reference:

MINISTRY OF HEALTH SINGAPORE. 2011. National Health Survey 2010. Singapore: Epidemiology and Disease Control Division, Ministry of Health Singapore. 